\title{
Identification of Small Presolar Spinel and Corundum Grains by Isotopic Raster Imaging
}

\author{
Ann Nguyen ${ }^{1}$, Ernst Zinner ${ }^{1}$ and Roy S. Lewis ${ }^{2}$ \\ ${ }^{1}$ Laboratory for Space Sciences, and the Physics Department, Washington University, \\ St Louis, MO 63130, USA \\ nguyen@levee.wustl.edu \\ ${ }^{2}$ Enrico Fermi Institute, University of Chicago, Chicago, IL 60637, USA
}

Received 2003 May 21, accepted 2003 September 21

\begin{abstract}
The oxygen isotopic compositions of densely packed submicron oxide grains in two grain separates of different grain size from the CM2 carbonaceous chondrite Murray were determined by multidetection raster imaging on the NanoSIMS ion microprobe. This led to the identification of 81 presolar spinel and 3 presolar corundum grains among $\sim 51700$ grains in the CF residue (mean diameter $0.15 \mu \mathrm{m}$ ) and 171 presolar spinel and 29 presolar corundum grains among $\sim 21500$ grains in the CG residue (mean diameter $0.45 \mu \mathrm{m}$ ). Previous NanoSIMS analysis of individual grains from the same residues has led to the discovery of 15 presolar spinel and 3 presolar corundum grains among 628 CF grains, and 9 presolar spinels among $753 \mathrm{CG}$ grains. The oxygen isotopic compositions of the presolar oxides found by raster imaging are comparable to those of the presolar oxides measured individually. While the abundance of presolar spinel among the (larger) grains of the CG residue is the same for both techniques, the detection efficiency for presolar spinel by imaging among the (smaller) grains in $\mathrm{CF}$ is lower due to the small size of these grains. Nonetheless, it is possible to identify presolar grains of this size range. Though single grain measurements are effective for determining the precise isotopic compositions and abundances of presolar grains, raster ion imaging is the method of choice in searches for rare presolar grain types such as presolar silicates.
\end{abstract}

Keywords: dust, extinction - meteors, meteoroids — methods: analytical — stars: AGB and post-AGB — nuclear reactions, nucleosynthesis, abundances

\section{Introduction}

Primitive meteorites contain presolar grains, tiny pieces of stardust that formed in the atmospheres of late-type stars and supernova ejecta. These grains survived interstellar travel, the formation of the solar system, and processes on the parent bodies of the meteorites in which they are found today. After extraction from meteorites they can be studied in detail in the laboratory (Zinner 1998). Their stellar origins are indicated by their isotopic compositions, which are completely different than those found in solar system materials. Among presolar grains identified to date are silicon carbide, graphite, silicon nitride, and oxides such as corundum $\left(\mathrm{Al}_{2} \mathrm{O}_{3}\right)$, spinel $\left(\mathrm{MgAl}_{2} \mathrm{O}_{4}\right)$, and hibonite $\left(\mathrm{CaAl}_{12} \mathrm{O}_{19}\right)$. Whereas almost all carbonaceous grains such as silicon carbide and graphite are of stellar origin, this is the case for only a small fraction of oxide grains. Presolar oxide grains were identified by isotopic anomalies found with the ion microprobe among the majority of isotopically normal grains of solar system origin. By far most presolar oxides discovered in the past were corundum (Nittler et al. 1997) and only a few presolar spinel grains had been found.

The residues CF and CG from the CM2 carbonaceous chondrite Murray contain mostly spinel grains and were prepared by Tang \& Anders (1988) through a series of physical and chemical separations that eliminated silicates, which dominate the composition of primitive meteorites. The average grain diameter of the $\mathrm{CF}$ residue is $0.15 \mu \mathrm{m}$, while that of the CG residue is $0.45 \mu \mathrm{m}$.
Throughout the paper, we make a distinction between these two residues because of differences in their grain size. This is important for placing a lower limit on the grain size that we can measure with the experimental technique applied and for determining the abundances of presolar grains as a function of grain size. Previous oxygen isotopic measurements of the $\mathrm{CF}$ fraction were made with the Cameca IMS $3 \mathrm{f}$ ion microprobe at Washington University (Zinner \& Tang 1988). These measurements had to be made on aggregates of many grains because, with an average grain size of $0.15 \mu \mathrm{m}$, the limited sensitivity of the ion probe did not allow for the analysis of individual $\mathrm{CF}$ grains. Excesses in ${ }^{17} \mathrm{O}$ provided a hint for the presence of presolar spinel in the $\mathrm{CF}$ residue but no unambiguous identification was achieved.

A new type of ion microprobe at Washington University, the Cameca NanoSIMS (Slodzian et al. 2003), allows for the measurement of oxygen isotopes in individual grains down to the size of Murray CF grains. This is made possible by the high secondary ion transmission at high mass resolution, the capacity for multidetection of isotopes, and the small primary ion beam size $(\sim 100 \mathrm{~nm})$, and thus high spatial resolution. With the NanoSIMS, we are able to apply two successful techniques to the analysis of submicron particles: single grain analysis of well dispersed grains, and raster ion imaging analysis of densely packed areas on a grain dispersion mount.

In the first technique well separated grains on a metal foil are identified from a raster image of secondary ions 
of an abundant isotope and/or secondary electrons. Subsequently, the primary ion beam is deflected onto individual grains within the rastered area and the isotopic compositions of these grains are measured one by one (thus single grain analysis). By deflecting the primary ion beam onto single grains within $20 \times 20 \mu \mathrm{m}^{2}$ areas on a gold foil, Zinner et al. (2003) used this technique to analyse individual Murray CF (average size $0.15 \mu \mathrm{m}$ ) and CG (average size $0.45 \mu \mathrm{m}$ ) grains for their $\mathrm{O}$ isotopic composition with the NanoSIMS. They identified 24 presolar spinels and found the abundances of presolar spinel grains among all (mostly spinel) grains in the $\mathrm{CF}$ and $\mathrm{CG}$ residues to be $2.4 \%$ and $1.2 \%$, respectively. This result and the known weight fractions of the $\mathrm{CF}$ and $\mathrm{CG}$ residues relative to the whole meteorite ( 28 and 45 ppm, respectively; Tang \& Anders 1988) established that presolar spinel constitutes at least $1 \mathrm{ppm}$ of the Murray carbonaceous meteorite.

While the analysis of single grains on well dispersed samples is effective for finding relatively abundant anomalous grains and for determining their precise isotopic compositions, raster ion imaging of tightly packed grains may be more efficient in detecting rare presolar grain types among the predominant isotopically normal grains. In this technique the primary beam is rastered over a certain area and digital images of the secondary ion counts of the isotopes of interest are obtained. In the NanoSIMS the beam is actually not rastered continuously but is stepped under computer control from one pixel location to the next within the rastered area. In the present study, we used multidetection raster imaging to measure the $\mathrm{O}$ isotopes of spinel grains from the Murray CF and CG residues. This analysis method had been employed by Messenger et al. (2003) in the discovery of presolar silicate grains in interplanetary dust particles. Thus, one of the motivations for this study is to optimise the isotopic imaging technique for the search for presolar silicate grains in primitive meteorites.

\section{Experimental}

Measurements were made on the same Murray CF and CG grain mounts that were used for single grain analysis (Zinner et al. 2003). These mounts were produced by dispensing grains from liquid suspension onto gold foils that had been pressed onto metal mounts. Also deposited onto the gold foil were $1 \mu \mathrm{m}$ terrestrial corundum grains and matrix grains from the carbonaceous chondrite Murchison, which served as standards. Most sample grains on the mounts are well separated from one another. These areas were chosen for single grain measurements. Areas on the sample mount that are almost completely covered with grains, however, were chosen for raster imaging. Figure 1 shows an area on the CG mount that contains both dispersed and tightly packed grains, demonstrating the difference in grain densities for the two types of analysis. For single grain measurements, there are typically 20-40 grains within a $20 \times 20 \mu \mathrm{m}^{2}$ area. On the other hand, when imaging is applied, there are up to $\sim 1600$ grains for $\mathrm{CG}$ and up to $\sim 15000$ grains for $\mathrm{CF}$ in an area of the same size.

The Cameca NanoSIMS ion microprobe at Washington University was used to make isotopic measurements in multidetection. A $\sim 150 \mathrm{~nm}, 16 \mathrm{keV} \mathrm{Cs}{ }^{+}$primary ion beam of $\sim 6 \mathrm{pA}$ intensity was rastered over $20 \times 20 \mu \mathrm{m}^{2}$, or in some cases $15 \times 15 \mu \mathrm{m}^{2}$, areas. For each area, between 20 and 40 sequential $256 \times 256$ pixel images were obtained to produce an integrated image. Because the linear size of one pixel $\left(78 \mathrm{~nm}\right.$ and $59 \mathrm{~nm}$ for $20 \times 20 \mu \mathrm{m}^{2}$ and $15 \times 15 \mu \mathrm{m}^{2}$ areas, respectively) is smaller than the primary beam diameter, the spatial resolution of the images is limited by the primary ion beam size. One complete image took between 3.5 and $7 \mathrm{~h}$, depending on the

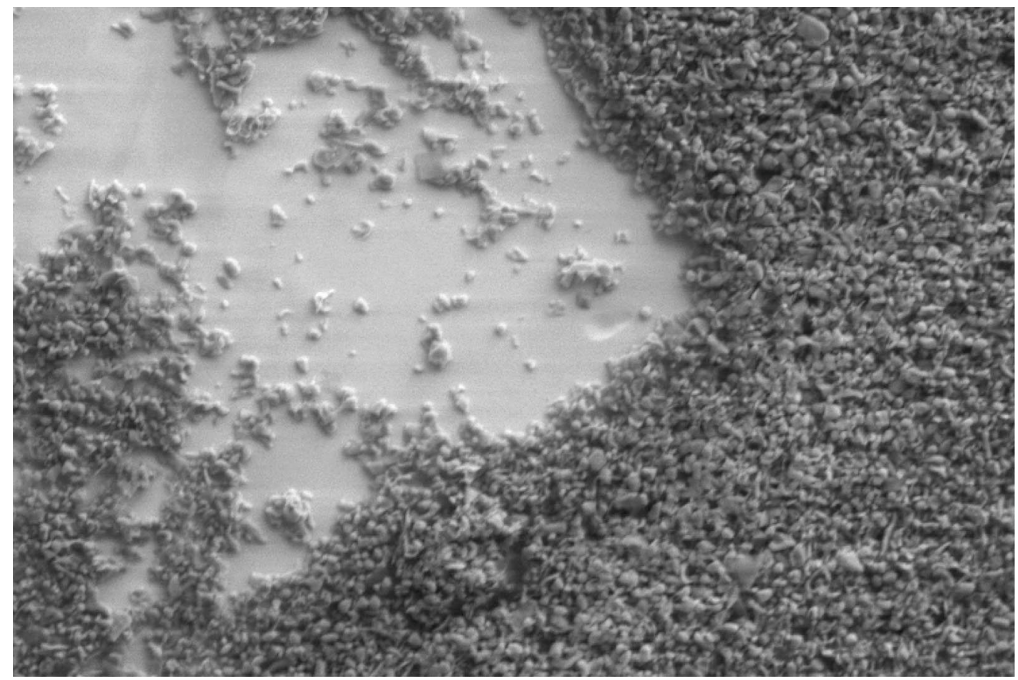

Figure 1 Secondary electron image of a $30 \times 20 \mu \mathrm{m}^{2}$ area on grain dispersion mount CG. The region in the centre contains well dispersed grains. Grains in such regions were used for single grain analysis. The region on the right is entirely covered with grains. Such regions were chosen for raster ion imaging. 
number of scans, and in this time less than $0.1 \mu \mathrm{m}$ of material was removed by the primary ion beam. While this depth is comparable to the size of some of the CF grains, and thus the smallest grains are sputtered away completely, useful information can still be obtained from them provided the number of counts is statistically large enough. As with the single grain measurements, negative secondary ions of the three $\mathrm{O}$ isotopes, ${ }^{24} \mathrm{Mg}^{16} \mathrm{O}$, and ${ }^{27} \mathrm{Al}^{16} \mathrm{O}$ were detected simultaneously in five small electron multipliers together with secondary electrons. The latter two ion species were detected in order to aid in the identification of the mineralogy of the analysed grains. It is important to note that, unlike previous imaging on the Cameca IMS $3 \mathrm{f}$ ion probe (Nittler et al. 1997), we are able to image ${ }^{17} \mathrm{O}^{-}$ (Figure 2). This capability is made possible by the high sensitivity achieved with the NanoSIMS at the high mass resolution required to separate the ${ }^{17} \mathrm{O}$ and ${ }^{16} \mathrm{OH}$ peaks. This sensitivity exceeds that obtained with the IMS $3 \mathrm{f}$ by at least a factor of 30 (up to 100 if the gain achieved by multidetection is taken into account).
Figure 2 shows oxygen isotopic images from one analysed area on the CG mount. The colour scale for the three isotopic images is adjusted to the average number of counts per pixel. Since most grains in the image are isotopically 'normal' (see below), the scale values are proportional to the isotopic abundances of the three $\mathrm{O}$ isotopes and the images of the three isotopes appear, disregarding statistical fluctuation, the same. The average number of ${ }^{16} \mathrm{O}$ counts per pixel for all images obtained is $3.2 \times 10^{4}$. Since the solar (i.e. terrestrial) ${ }^{17} \mathrm{O} /{ }^{16} \mathrm{O}$ ratio is $3.8 \times 10^{-4}$, one pixel contains, on average, $12{ }^{17} \mathrm{O}$ counts. Since the pixel size is smaller than the average grain size, the images were binned into bins of $2 \times 2$ pixels. Because one bin contains, on average, $\sim 49{ }^{17} \mathrm{O}$ counts, the counting (Poisson) statistical error on the ${ }^{17} \mathrm{O} /{ }^{16} \mathrm{O}$ ratio is $\sim 14 \%$ for each bin. For the ${ }^{18} \mathrm{O} /{ }^{16} \mathrm{O}$ ratio it is $\sim 6.3 \%$. These are also the average errors for $\mathrm{CF}$ grains whose average size of $0.15 \mu \mathrm{m}$ is comparable to the bin size for the $20 \times 20 \mu \mathrm{m}^{2}$ rastered area (for the $15 \times 15 \mu \mathrm{m}^{2}$ area they are somewhat smaller). For the
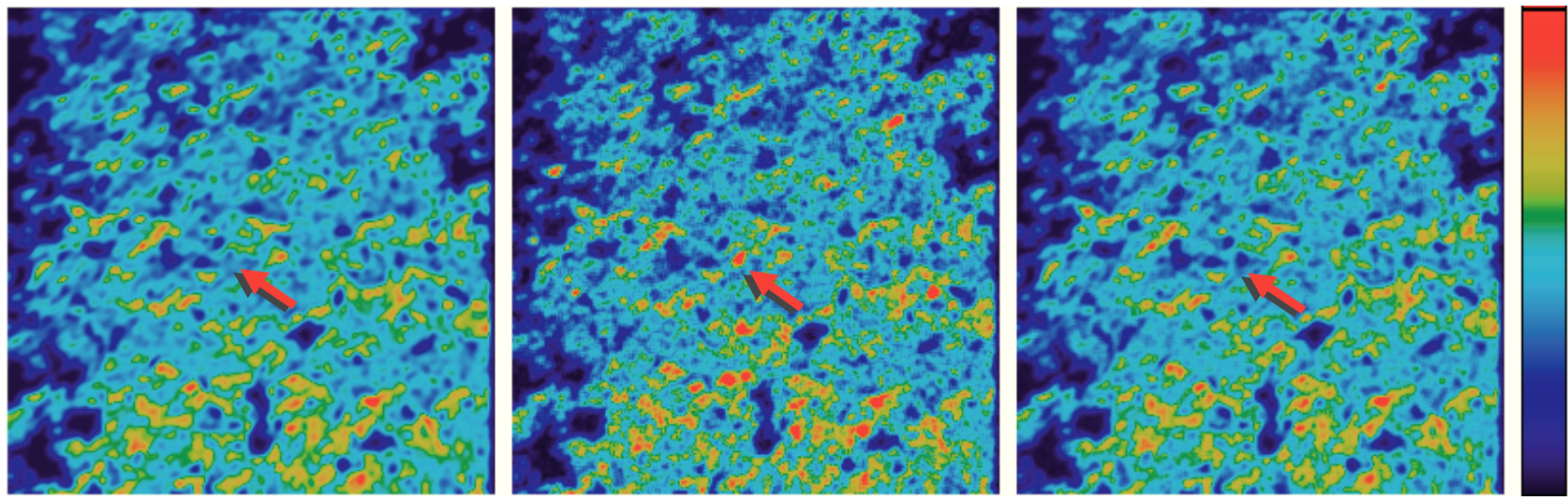

Figure $2{ }^{16} \mathrm{O}^{-},{ }^{17} \mathrm{O}^{-}$, and ${ }^{18} \mathrm{O}^{-}$ion images, shown in sequence, for a $20 \times 20 \mu \mathrm{m}^{2}$ area partially covered with Murray CG grains. The colour bar indicates relative intensity of the secondary ion signal, or number of counts per pixel. For each image it is scaled to the average number of counts per pixel and isotopically normal grains therefore have the same colour. Several anomalous grains are apparent in these images. The arrows point to a grain which is enriched in ${ }^{17} \mathrm{O}$ and depleted in ${ }^{18} \mathrm{O}$.
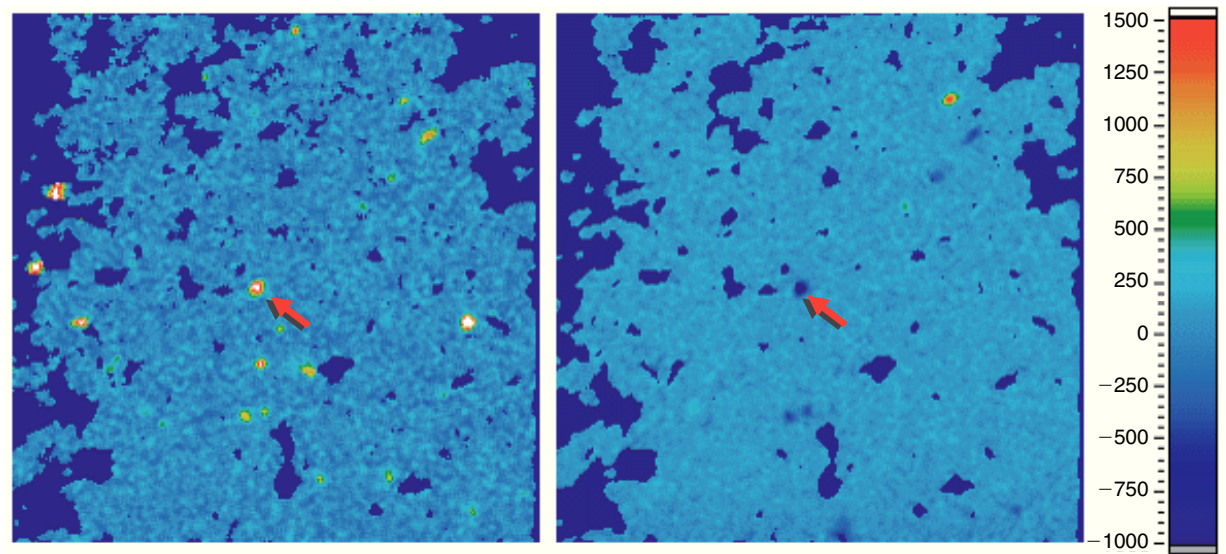

Figure $3{ }^{17} \mathrm{O} /{ }^{16} \mathrm{O}($ left $)$ and ${ }^{18} \mathrm{O} /{ }^{16} \mathrm{O}($ right $)$ ratio images for the same area shown in Figure 2 . The ratios are given as $\delta$-values, deviations from the SMOW ratio (see text) in per mille (\%o). Small scale variations are due to statistical fluctuations and are more apparent in the ${ }^{17} \mathrm{O} /{ }^{16} \mathrm{O}$ image because ${ }^{17} \mathrm{O}$ counts per pixel are lower than ${ }^{18} \mathrm{O}$ counts per pixel. Areas with large anomalies clearly indicate grains that are of presolar origin. The arrows point to the same anomalous grain as in Figure 2. 
larger CG grains of $0.45 \mu \mathrm{m}$ average diameter the errors on the isotopic ratios for a given grain are $4.9 \%$ and $2.2 \%$, respectively. Again, with the high sensitivity and thus low counting error afforded by the NanoSIMS, the ${ }^{17} \mathrm{O} /{ }^{16} \mathrm{O}$ isotopic ratios can be obtained directly from the ion images.

Isotopically anomalous grains can already be discerned in the isotopic images of Figure 2. The arrows in this figure point to a grain that is enriched in ${ }^{17} \mathrm{O}$ and depleted in ${ }^{18} \mathrm{O}$. Isotopically anomalous grains can be even more easily detected in isotopic ratio images (Figure 3 ). These images are given as $\delta$-values, deviations of the isotopic ratio from the standard O isotopic ratio of SMOW (standard mean ocean water) in per mille (\%o). For the normalisation we did not use an external standard but rather the isotopic ratios of the large majority of spinel grains on the mounts. This was done by assuming that the average $\mathrm{O}$ isotopic composition of the isotopically 'normal' grains (average over the whole image for each area) in the two residues is $\delta^{17} \mathrm{O}=\delta^{18} \mathrm{O}=-50 \%$ o relative to SMOW, typical for spinel grains in CM2 carbonaceous chondrites (Clayton \& Mayeda 1984). The results on grains from the CF and CG residues measured by the single grain analysis technique by Zinner et al. (2003), who used an external isotopic standard, showed that this is indeed the case. Although, on average, these grains differ in their $\mathrm{O}$ isotopic ratios by $-50 \%$ from SMOW, they are undoubtedly of solar system origin and we therefore call them and their isotopic compositions 'normal'.

Isotopically anomalous grains were identified from these ratio images and manually defined. The ${ }^{17} \mathrm{O} /{ }^{16} \mathrm{O}$ and ${ }^{18} \mathrm{O} /{ }^{16} \mathrm{O}$ ratios and statistical errors were then computed from the defined 'grain' area in the images. A grain was considered to be presolar if its isotopic composition differed by at least $3 \sigma$ from the composition of the isotopically 'normal' grains. Whereas anomalous grains were individually defined, isotopic ratios of normal grains were determined by dividing the regions in the images covered with grains into smaller areas of size comparable to the size of anomalous grains.

\section{Results}

One $15 \times 15 \mu \mathrm{m}^{2}$ image and four $20 \times 20 \mu \mathrm{m}^{2}$ images were taken for the Murray $\mathrm{CF}$ residue and fourteen $20 \times 20 \mu \mathrm{m}^{2}$ images for the Murray CG residue. We identified 81 presolar spinel grains and 3 presolar corundum grains for $\mathrm{CF}$ and 171 presolar spinel grains and 29 presolar corundum grains for CG. Spinel and corundum were identified from the $\mathrm{AlO}^{-} / \mathrm{MgO}^{-}$ratios. Their isotopic ratios fall in the range previously obtained for presolar corundum grains (Nittler et al. 1997) and are shown in Figure 4. The range for anomalous CF grains (mean size $0.15 \mu \mathrm{m})$, however, is not as broad as that for the CG grains (mean size $0.45 \mu \mathrm{m}$ ). This is undoubtedly due to the contribution of signal from surrounding grains of normal composition, which dilutes any anomalies in the small $\mathrm{CF}$ grains. While this dilution occurs with the CG grains as well, the effect is much smaller because these grains are on average 3 times larger than the CF grains.

In order to determine the abundance of presolar spinel and corundum grains found by raster imaging, we must first estimate the total number of analysed grains. This is done by determining the grain coverage for each image and by assuming that the covered areas are uniformly packed with grains of size $0.15 \mu \mathrm{m}$ and $0.45 \mu \mathrm{m}$ for Murray $\mathrm{CF}$

(a)

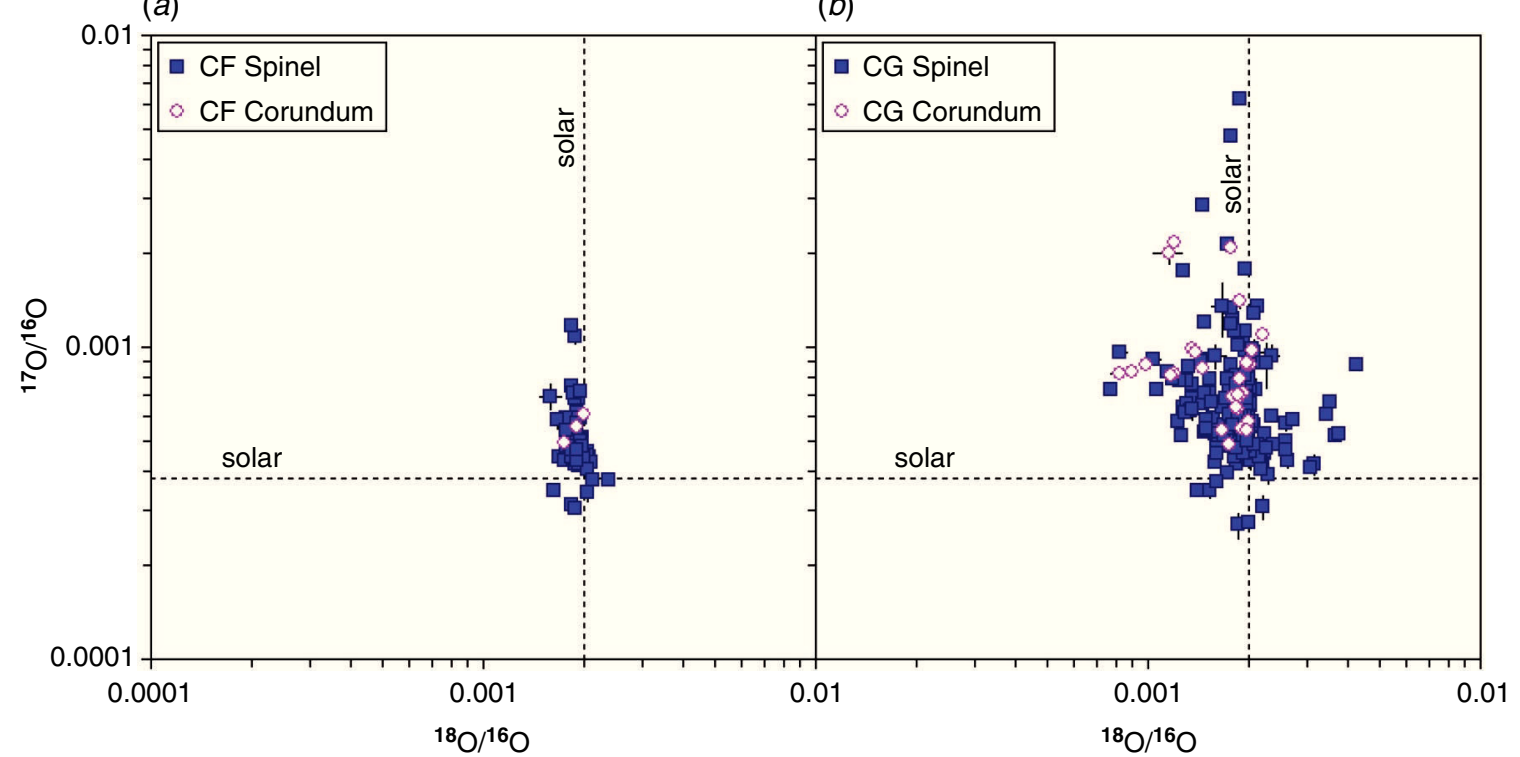

Figure 4 Oxygen isotopic ratios of presolar spinel and corundum from the Murray CF (mean size $0.15 \mu \mathrm{m})(a)$ and Murray CG (mean size $0.45 \mu \mathrm{m})(b)$ residues identified by raster ion imaging. Errors are given as $1 \sigma$. The dashed lines indicate solar ratios for ${ }^{17} \mathrm{O} /{ }^{16} \mathrm{O}(0.000383)$ and ${ }^{18} \mathrm{O} /{ }^{16} \mathrm{O}(0.002005)$. Although the $\mathrm{O}$ isotopic composition of the sun is not well known, in this and subsequent figures we use the term 'solar' for the composition of the solar system as found in meteoritic, lunar, and terrestrial samples, which, to first order, have identical $\mathrm{O}$ isotopic compositions. The above ratios are those of SMOW. 
Table 1. Fractions of presolar oxide grains in the Murray CF and CG residues as determined by raster ion imaging and single grain analysis and efficiencies of these measurements

\begin{tabular}{llcccccr}
\hline $\begin{array}{l}\text { Meteorite/ } \\
\text { residue }\end{array}$ & $\begin{array}{l}\text { Analysis } \\
\text { technique }\end{array}$ & $\begin{array}{c}\text { Presolar } \\
\text { spinel/total }\end{array}$ & $\begin{array}{c}\text { Presolar } \\
\text { corundum/total }\end{array}$ & $\begin{array}{c}\text { Abundance } \\
\text { spinel (\%) }\end{array}$ & $\begin{array}{c}\text { Abundance } \\
\text { corundum (\%) }\end{array}$ & $\begin{array}{c}\text { Grains } \\
\text { analysed/hour }\end{array}$ & $\begin{array}{c}\text { Presolar } \\
\text { grains found/hour }\end{array}$ \\
\hline Murray CF & Ion imaging & $81 / 51700$ & $3 / 51700$ & 0.2 & 0.01 & 2950 & 5 \\
$(0.15 \mu \mathrm{m})$ & Single grain* & $15 / 628$ & $3 / 628$ & 2.4 & 0.9 & 30 & $<1$ \\
Murray CG & Ion imaging & $171 / 21500$ & $29 / 21500$ & 0.8 & 0.1 & 410 & 4 \\
$(0.45 \mu \mathrm{m})$ & Single grain* & $9 / 753$ & $0 / 753$ & 1.2 & $<0.2$ & 30 & $<0.5$ \\
\hline
\end{tabular}

* From Zinner et al. (2003)

and $\mathrm{CG}$, respectively. We arrive at $\sim 51700 \mathrm{CF}$ grains analysed, for a presolar spinel abundance of $0.2 \%$ and a presolar corundum abundance of $0.01 \%$. An estimate of 21 500 analysed CG grains yields an abundance of $0.8 \%$ for presolar spinel and $0.1 \%$ for presolar corundum.

\section{Discussion}

The Murray CF and CG residues were specifically chosen to develop and test the raster ion imaging technique. One reason is that the abundances and $\mathrm{O}$ isotopic compositions of presolar oxide grains, as determined by Zinner et al. (2003), are known for both residues. In addition, the sizes of these grains are similar to the sizes of the presolar silicates found in interplanetary dust particles (Messenger et al. 2003). Thus, this study tests for the precision, spatial resolution, and efficiency of imaging measurements on densely packed grain areas.

One of the main features of raster ion imaging that makes it the method of choice for presolar grain searches is its ability to measure many more grains in a certain amount of time than individual grain analysis. In single grain mode, typically 30 grains per hour can be measured. It should be noted that this mode is not yet automated and automation will undoubtedly increase efficiency and make the constant presence of an operator unnecessary. Ion imaging of a $20 \times 20 \mu \mathrm{m}^{2}$ area with 20 scans takes $\sim 3.5 \mathrm{~h}$. Assuming ideal conditions, i.e. complete and uniform grain coverage of the area, $\sim 5000 \mathrm{CF}$ or $560 \mathrm{CG}$ grains can be measured in an hour. The efficiency achieved in this study is given in Table 1 and it is quite apparent that it is significantly greater than that achieved by single grain measurements.

The resultant abundances of presolar spinel and corundum for both ion imaging and previous single grain measurements are also summarised in the table. They are given as the percentage of presolar grains among all the grains analysed for each residue. Figure 5 shows the isotopic composition of the presolar spinel grains found in this study, as well as the 15 presolar Murray $\mathrm{CF}$ spinel grains and the 9 presolar Murray CG spinel grains that were previously measured individually (Zinner et al. 2003). The isotopic compositions determined by both methods of analysis are in agreement.

The presolar spinel abundance for Murray CF grains as determined by ion imaging is $0.2 \%$. In contrast,

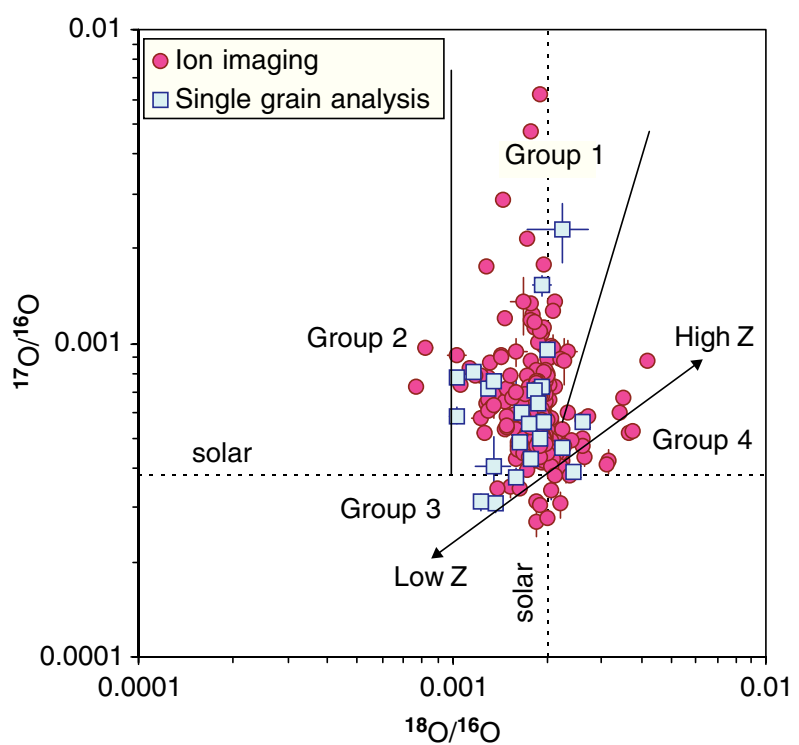

Figure 5 Oxygen isotopic ratios of the presolar spinel grains identified in this study by raster ion imaging, and the 24 spinels found by single grain analysis (Zinner et al. 2003). Data are for both the Murray CF and CG residues. Also shown are the regions of the four presolar oxide groups defined by Nittler et al. (1997), and a line with arrows indicating the Galactic evolution of $\mathrm{O}$ isotopes from low to high metallicity (Z) (Timmes et al. 1995).

the abundance from single grain measurements is $2.4 \%$ $(15 / 628)$. This discrepancy is certainly due to the small size of these grains (mean grain diameter $0.15 \mu \mathrm{m}$ ). During single grain measurements, only the signal from the grain of interest contributes to the isotopic ratios. On the other hand, with ion imaging, signal from the surrounding grains of normal composition will indubitably contribute to the isotopic ratios of an anomalous grain. Consequentially, the magnitude of anomalies will be reduced, and grains with relatively small anomalies appear to have normal composition. This also explains why anomalous CF spinel grains have a narrower range in their isotopic ratios than CF grains identified by single grain analysis and, as already mentioned, CG grains (mean grain diameter $0.45 \mu \mathrm{m}$ ). While imaging of CF grains may not be useful for the determination of their abundance and their exact isotopic ratios, it is a very effective means for identifying presolar grains in this size range.

For the Murray CG anomalous spinel grains, abundances of $0.8 \%$ and $1.2 \%$ were obtained from ion imaging 
and single grain analyses, respectively. The abundance from single grain measurements, however, is based on 9 presolar grains out of 753 measured grains and, within statistical error, the two abundances are in agreement.

The oxygen isotopic compositions of presolar oxide (mostly corundum) grains and their possible stellar sources have been discussed in some detail before and the interested reader is referred to the literature (Wasserburg, Boothroyd, \& Sackmann 1995; Nittler et al. 1997; Choi et al. 1998; Boothroyd \& Sackmann 1999; Nollett, Busso, \& Wasserburg 2003). There are grains present in all four groups of presolar oxides defined by Nittler (1997) among the grains of this study (Figure 5). Group 1 grains are believed to come from red giants or asymptotic giant branch stars whose $\mathrm{O}$ isotopic compositions are determined by the first (and second) dredge-up (Boothroyd \& Sackmann 1999). Group 2 grains most likely originated in low-mass stars that experienced extra mixing ('cool bottom processing') during which ${ }^{18} \mathrm{O}$ was destroyed (Wasserburg et al. 1995; Nollett et al. 2003). The ${ }^{16} \mathrm{O}$ excesses of Group 3 grains are not large enough to indicate a supernova ( $\mathrm{SN}$ ) origin. These grains have been interpreted to come from low-metallicity stars and their O ratios to reflect the Galactic evolution of the $\mathrm{O}$ isotopes (Timmes, Woosley, \& Weaver 1995). Group 4 grains, finally, could come from stars with higher-than-solar metallically, but those with the largest ${ }^{17} \mathrm{O}$ and ${ }^{18} \mathrm{O}$ excesses could have a SN origin (Choi et al. 1998).

Comparisons between presolar spinel grains and presolar corundum grains identified by single grain analysis have been discussed by Zinner et al. (2003). Figure 6 compares the isotopic ratios of the presolar spinel grains from Murray CG identified in this study and previously identified presolar corundum grains. The $\mathrm{CF}$ imaging results are not included because these isotopic ratios are more diluted than those of the CG grains.

In general, the oxygen isotopic ratio distributions of the presolar spinel and corundum grains are comparable. One glaring difference is that the spinel grains do not show the large ${ }^{18} \mathrm{O}$ depletions exhibited by corundum grains. Of course, dilution with isotopically normal $\mathrm{O}$ affects grains with depletions more than grains with excesses. On the other hand, the single grain measurements of spinel also did not reveal grains with large ${ }^{18} \mathrm{O}$ deficits (Zinner et al. 2003; see Figure 4), but limited statistics might have been the reason. Finally, there could be a grain size effect as most corundum grains plotted in Figure 6 were larger than $1 \mu \mathrm{m}$. Another difference is that the fraction of presolar corundum with large ${ }^{17} \mathrm{O}$ excesses is larger than that of spinel, indicating that more corundum grains come from stars with mass greater than $\sim 1.8 \mathrm{M}_{\odot}$ (Boothroyd \& Sackmann 1999). It appears also that the abundance of presolar spinel in Group $3\left({ }^{16} \mathrm{O}\right.$-rich grains) is smaller than that of corundum, and that the spinel grains in this group are not as ${ }^{16} \mathrm{O}$-rich as the corundum grains. On the other hand, there are more Group 4 spinel than corundum grains.

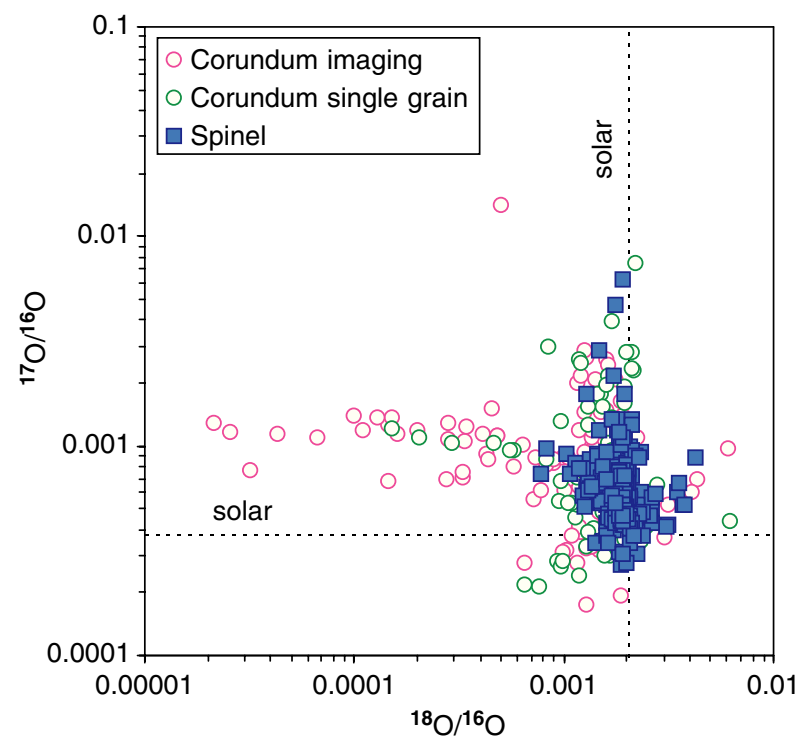

Figure 6 Oxygen isotopic ratios of presolar spinel grains from the Murray $\mathrm{CG}$ residue identified by raster ion imaging of all three $\mathrm{O}$ isotopes are compared to those of presolar corundum grains $>1 \mu \mathrm{m}$ identified by direct ion imaging of ${ }^{16} \mathrm{O}^{-}$and ${ }^{18} \mathrm{O}^{-}$on well dispersed grains (Nittler et al. 1997) and by single grain measurements. In general, the isotopic ratio distribution for the spinel grains is similar to that of the corundum grains but the range of isotopic ratios for spinel is smaller than that for corundum. Specifically, no spinels from this study show the large ${ }^{18} \mathrm{O}$ depletions exhibited by corundum grains (see text).

\section{Conclusions}

When we began this study, there were only 37 identified presolar spinel grains, and 26 of those had been measured individually on the Cameca NanoSIMS. Another method of oxygen isotopic analysis on the NanoSIMS, raster ion imaging of many grains in multidetection, can be extremely advantageous in the search for rare presolar grain types. In fact, ion imaging of the three oxygen isotopes has led to the discovery of presolar silicates in interplanetary dust particles (Messenger et al. 2003). We have performed isotopic analyses in which we imaged densely packed grains on the Murray CF and CG mounts used for single grain analysis. As a result, we have added 252 presolar spinel and 32 presolar corundum grains to the number of known presolar oxide grains. The oxygen isotopic compositions of these grains fall within the range of presolar corundum. While the abundance of presolar grains in Murray CF could not be reproduced due to the small grain size, it is important to note that we were able to identify anomalous grains from this residue. The abundance of the relatively larger Murray CG spinel grains was successfully reproduced. With its efficiency in the identification of presolar grains greater than $\sim 0.2 \mu \mathrm{m}$, raster ion imaging will play a key role in the search for presolar silicates in primitive meteorites.

\section{Acknowledgments}

We thank Tang Ming for preparing the Murray residues and are grateful to Scott Messenger and Frank Stadermann 
for developing the software for processing the isotopic raster images. Helpful comments by three reviewers are gratefully acknowledged. This work was supported by NASA grant NAG5-11545.

\section{References}

Boothroyd, A. I., \& Sackmann, I.-J. 1999, ApJ, 510, 232

Choi, B.-G., Huss, G. R., Wasserburg, G. J., \& Gallino, R. 1998, Science, 282, 1284

Clayton, R. N., \& Mayeda, T. K. 1984, E\&PSL, 67, 151

Messenger, S., Keller, L. P., Stadermann, F. J., Walker, R. M., \&

Zinner, E. 2003, Science, 300, 105
Nittler, L. R., Alexander, C. M. O. D., Gao, X., Walker, R. M., \& Zinner, E. 1997, ApJ, 483, 475

Nollett, K. M., Busso, M., \& Wasserburg, G. J. 2003, ApJ, 582, 1036 Slodzian, G., Hillion, F., Stadermann, F. J., \& Horreard, F. 2003, Applied Surface Science, 203/204, 798

Tang, M., \& Anders, E. 1988, GeCoA, 52, 1235

Timmes, F. X., Woosley, S. E., \& Weaver, T. A. 1995, ApJS, 98, 617 Wasserburg, G. J., Boothroyd, A. I., \& Sackmann, I.-J. 1995, ApJ, 447, L37

Zinner, E. 1998, AREPS, 26, 147

Zinner, E., \& Tang, M. 1988, LPI, 19, 1323

Zinner, E., Amari, S., Guinness, R., Nguyen, A., Stadermann, F., Walker, R. M., \& Lewis, R. S. 2003, GeCoA, in press 